\title{
LA SATISFACCIÓN SEXUAL EN PAREJAS CON ESTILOS DE APEGO SEGURO Y ANSIOSO
}

\author{
Isabel Chaves \\ ichavessayago@gmail.com \\ Lorena Caballero-Gascón \\ Roberta Ceccato \\ Vicente Morell-Mengual \\ M. Dolores Gil-Llario
}

Dpto. de Psicología Evolutiva y de la Educación. Universitat de València

Fecha de Recepción: 1 Marzo 2018

Fecha de Admisión: 10 Abril 2018

\section{RESUMEN}

Debido a la fuerte vinculación entre la satisfacción sexual, de pareja y el apego de ambos miembros de la diada, el objetivo de esta investigación fue examinar la satisfacción de pareja y sexual en las diferentes combinaciones de estilos de apego. Para ello se analizó la satisfacción sexual y de pareja en los diferentes estilos de apego de cada participante y de su pareja. En el estudio participaron 86 parejas heterosexuales, con edades entre los 20 y 60 años. Los resultados muestran que las personas seguras tienen una mayor satisfacción sexual y de pareja. Las diadas constituidas por un hombre seguro y una mujer segura son las que más satisfechas están, las mujeres seguras están menos satisfechas con sus parejas cuando ellos son preocupados pero los hombres seguros se encuentran igualmente satisfechos tanto si las mujeres son seguras como si son ansiosas. Por tanto, la satisfacción de un miembro de la pareja ansioso/a va a depender del estilo de apego y del sexo de su pareja.

Palabras clave: estilos de apego; parejas; satisfacción de pareja y satisfacción sexual

\section{ABSTRACT}

Sexual satisfaction in couples with secure or anxious attachment styles.

Due to the strong link between sexual satisfaction, relational satisfaction and the attachment of both members of the dyad, the aim of this study was to examine the sexual and relational satisfaction in different attachment styles combinations. To address this, sexual and relational satisfaction in the different attachment styles of each participant as well as their partners were analyzed. Eightysix heterosexual couples participated, aged 20-60 years old. The results show that people with secure attachment have both better sexual and relational satisfaction. Heterosexual couples where both are secure, are those who are most satisfied. Secure women are less satisfied with their cou- 


\section{LA SATISFACCIÓN SEXUAL EN PAREJAS CON ESTILOS DE APEGO SEGURO Y ANSIOSO}

ple when men are anxious. However, men are equally satisfied whether the women feel secure or anxious. Thus, the satisfaction of the anxious member of the couple will depend on the attachment styles as well as the sex of the partner.

Keywords: attatchmen styles; dyad; relational satisfaction and sexual satisfaction

\section{ANTECEDENTES}

La extensión de la Teoría del Apego a las relaciones afectivas adultas ha promovido un marco de indudable valor para estudiar las relaciones de pareja. Actualmente son muy escasos los estudios que evalúan la experiencia del apego en ambos miembros de la pareja, siendo más habitual una evaluación individual. La mayoría de ellos se han realizado en países como Estados Unidos, Canadá y Australia, siendo poco frecuentes en España (Martínez-Álvarez, Fuertes-Martín, Orgaz, Vicario y González-Ortega, 2014; Molero, Shaver, Ferrer, Cuadrado y Alonso-Arbiol, 2011). Y lo mismo ocurre con la satisfacción sexual (Rehman, Rellini y Fallis, 2011).

La Teoría del Apego (Bowlby, 1969/82) señala que la pareja está compuesta por la interacción de tres sistemas de conductas, el sistema de apego, el sistema sexual y el sistema de cuidados. El buen funcionamiento de cada uno de ellos favorece el mantenimiento de vínculos afectivos satisfactorios (Birnbaum, Reis, Mikulincer, Gillath, Orpaz, 2006; Gómez-Zapiain, Ortiz y Gómez-Lope, 2011, Melero, 2008). Hollan, Fraley y Roisman (2012) señalan que el apego seguro es un factor muy importante para que la relación de pareja se mantenga estable. Banse (2004) afirma que la satisfacción de pareja se asocia con el estilo de apego de cada miembro de la diada y con la combinación de ambos. Según Magai (1999) los patrones de emparejamiento más probables son: que los dos miembros de la pareja sean seguros; que uno sea seguro y el otro preocupado; que uno sea seguro y el otro huidizo; y que uno sea huidizo y el otro preocupado, siendo menos común la pareja preocupado-preocupado o huidizo-huidizo. Cuando ambos son seguros informan de mayor satisfacción con la pareja que cuando uno es seguro y otro inseguro o los dos son inseguros (0ttu y Akpan, 2011). Turan y Vicary (2010) constatan que los preocupados/ansiosos no valoran el apoyo que les puede ofrecer una persona con un apego seguro, siendo escasa la diferencia de satisfacción en ellos cuando están con un individuo seguro o inseguro. Esto les lleva a considerar que la relación entre el estilo de apego y la satisfacción de pareja está regulada por la conducta percibida del otro. Por el contrario, Cohn, Silver, Cowan y Pearson (1992) no encuentran diferencias entre las parejas formadas por dos seguros y las parejas mixtas (seguro-inseguro) considerando que el apego seguro puede actuar como amortiguador de los efectos negativos introducidos por el miembro inseguro de la relación. Por lo tanto, la satisfacción de una persona insegura va a depender de la conducta que perciba de su pareja, haciendo que puedan o no haber diferencias entre las parejas seguras y mixtas. Hasta el momento las investigaciones realizadas en este sentido no han sido concluyentes, tanto por su escasez como por utilizar procedimientos de emparejamiento hipotético (Lafuente y Cantero, 2010).

En lo que respecta a la satisfacción sexual, las personas seguras tienen mayor satisfacción que las ansiosas. La comodidad en la intimidad y el disfrute en las interacciones sexuales contribuyen en sus relaciones sentimentales estables y satisfactorias. Experimentan menos emociones negativas y más emociones positivas y apasionadas durante la actividad sexual que los inseguros y presentan mayor placer en el uso del tacto para expresar el afecto y la sexualidad. Los preocupados manifiestan menor satisfacción en tanto que la conducta sexual está relacionada con la búsqueda de proximidad. Utilizan el sexo como estrategia para mantener implicada a la pareja en la relación, buscar su aprobación y alcanzar la intimidad emocional y física (Birnbaum et al., 2006; Gómez-Zapiain et al., 2011; Rivera, Cruz, y Muñoz, 2011). MacNeil y Byers $(1997,2005)$ propusieron dos vías, la 
expresiva y la instrumental. Ambas vías postulan que la comunicación abierta sobre el sexo con la pareja contribuye a una mayor satisfacción sexual (Montesi, Conner, Gordon, Fauber, Kim y Heimberg, 2013). La satisfacción de pareja, por su parte, según la Teoría de la Satisfacción Marital y el Modelo Multidimensional de Pick y Andrade (1988) se centra en las actitudes que prevalecen hacia la interacción diádica y los aspectos de la pareja (Armenta, Sánchez, Díaz, 2014; Vera, 2010).

Las mujeres cuya pareja puntúa alto en sensibilidad interpersonal tienden a estar más satisfechas; sin embargo, la sensibilidad interpersonal de las mujeres no afecta a la satisfacción de los hombres (Bradbury, Campbell y Fincham, 1995). Por tanto, la relación entre la satisfacción marital y la interacción entre los miembros de la diada es más importante para las mujeres que para los hombres (Schmitt, Kliegel y Shapiro, 2007). Como señala Vera (2010) existe una fuerte vinculación entre estos tres constructos, la satisfacción sexual, la satisfacción de pareja y el estilo de apego de ambos miembros, lo que nos lleva a la necesidad de analizarlos conjuntamente, estudiando la satisfacción de pareja y sexual en las diferentes combinaciones de estilos de apego que forman las parejas.

\section{MÉTODO}

\section{Participantes}

En el estudio participaron 172 personas, 86 parejas formadas por un hombre y una mujer. El requisito para participar en este estudio era tener una pareja estable, es decir, con una duración igual o superior a dos años. Este tiempo es el mínimo necesario para que la relación se considere estable y haya tenido lugar la formación y consolidación de un vínculo de apego entre los miembros de la pareja (Hazan y Zeifman, 1994).

La edad promedio de los participantes es de 37.08 (SD=12.19) con un mínimo de 20 años y un máximo de 60 . El $45.3 \%$ tienen entre $25-35$ años, el $25.6 \%$ entre $36-45$ años y el $29.1 \%$ se encuentran entre los 46 y los 60 años. La media de años de relación estable con la pareja es de 15.94 ( $S D=12.12)$ con un mínimo de 2 años y un máximo de 43. El 4.7\% Ilevan 2 años de relación, el $20.9 \%$ entre 2.1 y 4 años, el $16.3 \%$ entre 4.1 y 8 años y el $58.1 \%$ llevan más de 8 años. Por lo que se refiere al tipo de apego, el $73.8 \%$ tienen un estilo de apego seguro y el $26.2 \%$ un apego ansioso.

\section{Instrumentos}

Experiences in Close Relationships-Revised (ECR-R; Fraley, Waller y Brennan, 2000)._Adaptado por Fernández-Fuertes, Orgaz y Carcedo, 2011._Consta de 18 ítems y se responde mediante una escala tipo Likert que oscila de 1 (Totalmente en desacuerdo) a 7 (Totalmente de acuerdo). Evalúa dos dimensiones del apego en una relación de pareja: la dimensión de ansiedad (9 ítems) y la dimensión de evitación (9 ítems). El alpha de Cronbach en cada subescala es de .80 y .86, respectivamente. El resultado se obtiene promediando la puntuación de los 9 ítems obtenidos en cada dimensión; una mayor puntuación indica mayor ansiedad y/o evitación. Utilizando estas dos subescalas se obtienen los estilos de apego adulto: un estilo de apego seguro, asociado a bajos niveles de ansiedad y evitación y tres estilos inseguros: preocupado (alta ansiedad y baja evitación), alejado (baja ansiedad y alta evitación) y temeroso (alta ansiedad y alta evitación).

Escala de Satisfacción Marital (ESM) (Pick y Andrade, 1988)_Esta escala está compuesta por 24 ítems, cada uno de ellos tiene tres opciones de respuesta que permiten expresar el nivel de satisfacción del individuo, respecto a lo que se plantea en la afirmación: 1= me gustaría muy diferente, $2=$ me gustaría algo diferente y $3=$ me gusta cómo está pasando. Evalúa tres dimensiones, la dimensión satisfacción en la interacción con la pareja (ítems 1-10), la satisfacción con los aspectos emocionales de la pareja (ítems 11-15) y la satisfacción con la organización y estructuración de la rela- 


\section{LA SATISFACCIÓN SEXUAL EN PAREJAS CON ESTILOS DE APEGO SEGURO Y ANSIOSO}

ción (ítems 16-24). El punto de corte es 48, el valor mínimo 24 puntos y el máximo 72, de modo que cuanto mayor sea la satisfacción con la pareja mayor será la puntuación. El Alpha de Cronbach de cada factor es $.90, .81$ y .85 .

Escala de Satisfacción Sexual (Rodríguez, 2010). Consta de 33 ítems, con una escala de respuesta que va desde 1 (siempre) hasta 4 (nunca). Evalúa la reacción emocional y la comunicación. El resultado en cada escala se obtiene promediando la puntuación de los 17 ítems de reacción emocional y los 16 de comunicación. El Alpha de Cronbach es de .92.

\section{Procedimiento}

Para recoger la muestra se contactó, vía correo electrónico, con diversas asociaciones españolas ofreciéndoles información sobre el estudio que nos disponíamos a desarrollar y solicitándoles su colaboración. Estas asociaciones divulgaron, a través de sus páginas web y/o sus redes sociales, un texto que contenía una breve explicación sobre el estudio y un enlace que remitía a los cuestionarios online. Cuando el participante accedía a la encuesta online se le proporcionaba una información más detallada sobre los objetivos del estudio y sobre el tratamiento estadístico de la información proporcionada, y se obtenía su consentimiento informado. El tiempo requerido para cumplimentar la batería fue de 80-90 minutos.

\section{RESULTADOS}

El primer nivel de análisis consistió en comparar la satisfacción sexual y de pareja según el estilo personal de apego, fuere cual fuere el de la pareja.

Como podemos observar en la tabla 1, tanto la satisfacción sexual como la satisfacción de pareja son superiores en las personas seguras en comparación con el de las personas con apego preocupado. Existen diferencias significativas a favor de las personas seguras en el primer factor de satisfacción de pareja, esto es, la satisfacción en la interacción con la pareja ( $\mathrm{t}=3.439, \mathrm{p}<.001)$, en el segundo factor, la satisfacción con los aspectos emocionales de la pareja $(\mathrm{t}=3.119, \mathrm{p}<.002)$, en el tercer factor, la satisfacción con la organización y estructuración de la relación ( $\mathrm{t}=3.390, \mathrm{p}<.001)$ y en la puntuación total, la satisfacción con la pareja ( $\mathrm{t}=3.827, \mathrm{p}<.000)$. Sólo en la comunicación se han encontrado diferencias significativas ( $\mathrm{t}=-1.953, \mathrm{p} \geq .005)$ a favor de los preocupados.

Tabla 1. Comparación en cuanto a la satisfacción de pareja y sexual en función de los 2 estilos de apego

\begin{tabular}{|c|c|c|c|c|}
\hline & $\begin{array}{c}\text { Apego } \\
\text { seguro } \\
\text { Media } \\
\text { (DT) } \\
\end{array}$ & $\begin{array}{c}\text { Apego } \\
\text { preocupado } \\
\text { Media } \\
\text { (DT) }\end{array}$ & $t$ & $p$ \\
\hline Satisfacción interacción pareja & $\begin{array}{l}25.28 \\
(3.10)\end{array}$ & $\begin{array}{l}23.42 \\
(3.17)\end{array}$ & 3.439 & .001 \\
\hline Satisfacción emocional pareja & $\begin{array}{l}12.58 \\
(1.75)\end{array}$ & $\begin{array}{l}11.62 \\
(1.84)\end{array}$ & 3.119 & .002 \\
\hline Satisfacción organización y estructuración relación & $\begin{array}{l}22.92 \\
(2.67)\end{array}$ & $\begin{array}{l}21.20 \\
(3.55)\end{array}$ & 3.390 & .001 \\
\hline Satisfacción pareja (P.Total) & $\begin{array}{l}60.79 \\
(6.54)\end{array}$ & $\begin{array}{l}56.24 \\
(7.66)\end{array}$ & 3.827 & .000 \\
\hline Satisfacción sexual: Reacción emocional & $\begin{array}{l}24.08 \\
(4.65)\end{array}$ & $\begin{array}{l}23.71 \\
(4.70)\end{array}$ & .454 & .650 \\
\hline Satisfacción sexual: Comunicación & $\begin{array}{l}26.33 \\
(6.11)\end{array}$ & $\begin{array}{l}28.47 \\
(6.82)\end{array}$ & -1.953 & .052 \\
\hline
\end{tabular}


En segundo lugar se analizó si existían diferencias en cuanto a la satisfacción de pareja y sexual entre los hombres y las mujeres en las siguientes combinaciones de apego: (1) hombre seguromujer segura; (2) hombre seguro-mujer preocupada; (3) hombre preocupado-mujer segura; y (4) hombre preocupado-mujer preocupada.

Como podemos observer en la tabla 2 las mujeres están más satisfechas con su pareja cuando ambos son seguros que en el resto de combinaciones. Las mujeres seguras tienen una mayor satisfacción en la interacción con la pareja cuando él es seguro $(\mathrm{M}=25.77$; $\mathrm{DT}=2.76)$ que cuando es preocupado ( $M=22.10$; $D T=2.84)$, siendo significativa esta relación $(F=6.232, p<.001)$. Las mujeres preocupadas tienen mayor satisfacción en la interacción con la pareja si él es preocupado $(M=24.45$; $\mathrm{DT}=1.04)$ que si es seguro $(\mathrm{M}=23.38$; $\mathrm{DT}=3.89)$, si bien, tales diferencias no son significativas.

Las mujeres seguras también tienen una mayor satisfacción con los aspectos emocionales de la pareja cuando él es seguro $(M=13.08, D T=1.38)$ que cuando es preocupado $(M=11.80, D T=1.99)$, aunque estas diferencias no son significativas. Las mujeres preocupadas tienen una mayor satisfacción con los aspectos emocionales de la pareja cuando él es preocupado ( $\mathrm{M}=11.73, \mathrm{DT}=1.27)$ que cuando es seguro ( $M=11.69, \mathrm{DT}=2.02)$, dichas diferencias no son significativas.

Las mujeres seguras tienen una mayor satisfacción en la organización y estructuración de la relación cuando él también es seguro $\quad(M=23.44 ; \quad D T=2.26)$ que si es preocupado $(M=20.50$; $\mathrm{DT}=3.34)$, esta relación es estadísticamente significativa $(\mathrm{F}=4.094, \mathrm{p}<.009)$. Las mujeres preocupadas tienen una mayor satisfacción en la organización y estructuración de la relación cuando él es seguro ( $M=22.46$, $D T=3.69)$ que cuando es preocupado $(M=21.91$, $D T=1.97)$, no siendo significativas estas diferencias.

Hay diferencias significativas $(\mathrm{F}=6.349, \mathrm{p}<.001)$ en la satisfacción global con la pareja cuando ambos son seguros $(M=62.29$; $D T=5.68)$ que cuando ella es segura y él es preocupado $(M=54.40$; $\mathrm{DT}=6.74)$. Las preocupadas tienen una mayor satisfacción si el hombre es preocupado $(\mathrm{M}=58.09$, $\mathrm{DT}=3.59)$ que si es seguro $(\mathrm{M}=57.57, \mathrm{DT}=8.41)$, si bien tales diferencias no son significativas.

Tabla 2. Comparación de medias en la satisfacción de pareja y sexual en las mujeres en las diferentes combinaciones de apego con sus parejas

\begin{tabular}{|c|c|c|c|c|c|c|c|}
\hline & $\begin{array}{c}\text { Seguro- } \\
\text { segura } \\
\text { Media } \\
\text { (DT) }\end{array}$ & $\begin{array}{c}\text { Seguro- } \\
\text { Preocupado } \\
\text { Media } \\
\text { (DT) } \\
\end{array}$ & $\begin{array}{c}\text { Preocupado- } \\
\text { Segura } \\
\text { Media } \\
\text { (DT) } \\
\end{array}$ & $\begin{array}{c}\text { Preocupado- } \\
\text { Preocupada } \\
\text { Media } \\
\text { (DT) } \\
\end{array}$ & $\boldsymbol{F}$ & $p$ & Bonferroni \\
\hline $\begin{array}{l}\text { Satisfacción } \\
\text { interacción } \\
\text { pareja }\end{array}$ & $\begin{array}{l}25.77 \\
(2.76)\end{array}$ & $\begin{array}{l}23.38 \\
(3.88)\end{array}$ & $\begin{array}{l}22.10 \\
(2.84)\end{array}$ & $\begin{array}{l}24.45 \\
(1.04)\end{array}$ & 6.232 & .001 & $\begin{array}{l}1>2 \\
1>3\end{array}$ \\
\hline $\begin{array}{l}\text { Satisfacción } \\
\text { emocional } \\
\text { pareja }\end{array}$ & $\begin{array}{l}13.08 \\
(1.38)\end{array}$ & $\begin{array}{l}11.69 \\
(2.02)\end{array}$ & $\begin{array}{l}11.80 \\
(1.99)\end{array}$ & $\begin{array}{l}11.73 \\
(1.27)\end{array}$ & 5.111 & .003 & $1>2$ \\
\hline $\begin{array}{l}\text { Satisfacción } \\
\text { organización y } \\
\text { estructuración } \\
\text { relación }\end{array}$ & $\begin{array}{l}23.44 \\
(2.26)\end{array}$ & $\begin{array}{l}22.46 \\
(3.69)\end{array}$ & $\begin{array}{l}20.50 \\
(3.34)\end{array}$ & $\begin{array}{l}21.91 \\
(1.97)\end{array}$ & 4.094 & .009 & $1>3$ \\
\hline $\begin{array}{l}\text { Satisfacción } \\
\text { pareja (Total) }\end{array}$ & $\begin{array}{l}62.29 \\
(5.68)\end{array}$ & $\begin{array}{l}57.54 \\
(8.41)\end{array}$ & $\begin{array}{l}54.40 \\
(6.74)\end{array}$ & $\begin{array}{l}58.09 \\
(3.59)\end{array}$ & 6.349 & .001 & $1>3$ \\
\hline $\begin{array}{l}\text { Satisfacción } \\
\text { sexual: } \\
\text { Reacción } \\
\text { emocional }\end{array}$ & $\begin{array}{l}24.64 \\
(4.53)\end{array}$ & $\begin{array}{l}23.85 \\
(4.69)\end{array}$ & $\begin{array}{l}24.20 \\
(4.98)\end{array}$ & $\begin{array}{l}24.09 \\
(5.78)\end{array}$ & 1.251 & .297 & \\
\hline $\begin{array}{l}\text { Satisfacción } \\
\text { sexual: } \\
\text { Comunicación }\end{array}$ & $\begin{array}{l}26.25 \\
(5.03)\end{array}$ & $\begin{array}{l}27.23 \\
(9.58)\end{array}$ & $\begin{array}{l}29.80 \\
(9.00)\end{array}$ & $\begin{array}{l}29.09 \\
(4.89)\end{array}$ & .136 & .938 & \\
\hline
\end{tabular}




\section{LA SATISFACCIÓN SEXUAL EN PAREJAS CON ESTILOS DE APEGO SEGURO Y ANSIOSO}

Finalmente, observamos que los hombres están más satisfechos con sus parejas cuando ambos son seguros que cuando ambos son preocupados (ver Tabla 3). Los hombres tienen una mayor satisfacción en la interacción con la pareja cuando ellos son seguros, independientemente de que ella sea segura ( $M=25.38, D T=3.18)$ o preocupada $(M=25.38$, $D T=3.18)$. Por otro lado, los hombres preocupados tienen una mayor satisfacción en la interacción con la pareja si ella es segura $(\mathrm{M}=23.40, \mathrm{DT}=2.80)$ que si es preocupada $(\mathrm{M}=22.45$, $\mathrm{DT}=3.98)$, no siendo significativas estas diferencias.

Los hombres tienen una mayor satisfacción con los aspectos emocionales de la pareja cuando son seguros y ella también los es $(M=12.42$, $D T=1.85)$ que cuando la mujer es preocupada $(\mathrm{M}=11.85, \mathrm{DT}=2.08)$, estas diferencias no son significativas. Los hombres preocupados tienen una mayor satisfacción con los aspectos emocionales de la pareja si la mujer es segura ( $M=12.40$, $\mathrm{DT}=1.51)$ que si es preocupada $(\mathrm{M}=10.73$, $\mathrm{DT}=2.20)$ aunque estas diferencias no son significativas.

Ellos presentan una mayor satisfacción con la organización y estructuración de la relación cuando son seguros y la mujer es preocupada $(N=23.00, D T=2.04)$ que cuando ella es segura $(M=22.85$, $\mathrm{DT}=2.86$ ), dichas diferencias no son significativas. Los hombres preocupados tienen una mayor satisfacción con la organización y estructuración de la relación si la mujer es segura ( $\mathrm{M}=20.30$, $\mathrm{DT}=2.71)$ que si es preocupada $(\mathrm{M}=19.82$, $\mathrm{DT}=4.81)$, si bien dichas diferencias no son significativas.

Los hombres seguros tienen una mayor satisfacción global de pareja si ella es segura ( $M=60.65$, $\mathrm{DT}=6.79)$ que si es preocupada $(\mathrm{M}=60.23, \mathrm{DT}=6.10)$, estas diferencias no son significativas. Los hombres preocupados tienen una mayor satisfacción global de pareja cuando la mujer es segura $(\mathrm{M}=56.10, \mathrm{DT}=6.05)$ que cuando es preocupada $(\mathrm{M}=53.00, \mathrm{DT}=10.48)$, aunque estas diferencias no son significativas.

Tabla 3. Comparación de medias en la satisfacción de pareja y sexual de los hombres en las diferentes combinaciones de apego con sus parejas

\begin{tabular}{|c|c|c|c|c|c|c|}
\hline & $\begin{array}{c}\text { Seguro- } \\
\text { segura } \\
\text { Media } \\
\text { (DT) } \\
\end{array}$ & $\begin{array}{l}\text { Seguro- } \\
\text { Preocupado } \\
\text { Media } \\
\text { (DT) } \\
\end{array}$ & $\begin{array}{c}\text { Preocupado- } \\
\text { Segura } \\
\text { Media } \\
\text { (DT) } \\
\end{array}$ & $\begin{array}{l}\text { Preocupado- } \\
\text { Preocupada } \\
\text { Media } \\
\text { (DT) }\end{array}$ & $F$ & $p$ \\
\hline $\begin{array}{l}\text { Satisfacción } \\
\text { interacción pareja }\end{array}$ & $\begin{array}{l}25.38 \\
(3.18)\end{array}$ & $\begin{array}{l}25.38 \\
(3.18)\end{array}$ & $\begin{array}{l}23.40 \\
(2.80)\end{array}$ & $\begin{array}{l}22.45 \\
(3.98)\end{array}$ & 3.234 & .026 \\
\hline $\begin{array}{l}\text { Satisfacción emocional } \\
\text { pareja }\end{array}$ & $\begin{array}{l}12.42 \\
(1.85)\end{array}$ & $\begin{array}{l}11.85 \\
(2.08)\end{array}$ & $\begin{array}{l}12.40 \\
(1.51)\end{array}$ & $\begin{array}{l}10.73 \\
(2.20)\end{array}$ & 2.583 & .059 \\
\hline $\begin{array}{l}\text { Satisfacción } \\
\text { organización y } \\
\text { estructuración } \\
\text { relación }\end{array}$ & $\begin{array}{l}22.85 \\
(2.86)\end{array}$ & $\begin{array}{l}23.00 \\
(2.04)\end{array}$ & $\begin{array}{l}20.30 \\
(2.71)\end{array}$ & $\begin{array}{l}19.82 \\
(4.81)\end{array}$ & 4.595 & .005 \\
\hline $\begin{array}{l}\text { Puntuación total: } \\
\text { Satisfacción pareja }\end{array}$ & $\begin{array}{l}60.65 \\
(6.79)\end{array}$ & $\begin{array}{l}60.23 \\
(6.10)\end{array}$ & $\begin{array}{l}56.10 \\
(6.05)\end{array}$ & $\begin{array}{c}53.00 \\
(10.48)\end{array}$ & 4.148 & .009 \\
\hline $\begin{array}{l}\text { Satisfacción sexual: } \\
\text { Reacción emocional }\end{array}$ & $\begin{array}{l}23.73 \\
(4.79)\end{array}$ & $\begin{array}{l}23.00 \\
(4.53)\end{array}$ & $\begin{array}{l}24.80 \\
(4.85)\end{array}$ & $\begin{array}{l}22.18 \\
(3.55)\end{array}$ & 0652 & .584 \\
\hline $\begin{array}{l}\text { Satisfacción sexual: } \\
\text { Comunicación }\end{array}$ & $\begin{array}{l}26.04 \\
(6.08)\end{array}$ & $\begin{array}{l}26.54 \\
(7.98)\end{array}$ & $\begin{array}{l}29.90 \\
(6.33)\end{array}$ & $\begin{array}{l}28.09 \\
(5.34)\end{array}$ & 1.208 & .312 \\
\hline
\end{tabular}




\section{DISCUSIÓN}

El objetivo de este trabajo ha sido estudiar la satisfacción de pareja y la satisfacción sexual en las diferentes combinaciones de estilos de apego que forman las diadas. Nuestros datos apuntan, en la línea de los obtenidos por Alexandrov, Cowan, y Cowan, (2005); Bernier y Dozier, (2002); Hazan y Shaver (1987); Mikulincer y Florian, (1999), a que las personas seguras, independientemente del estilo de apego del otro miembro de la pareja, tienen mayor satisfacción sexual y de pareja que las preocupadas. Por un lado, los seguros interactúan mejor con su pareja, están satisfechos con las reacciones emocionales del otro, se organizan y estructuran mejor y tienen una mayor satisfacción con la pareja en general, que los ansiosos. Por otro, se encuentran más cómodos en la intimidad y en las interacciones sexuales y experimentan menos emociones negativas y más emociones positivas y apasionadas durante la actividad sexual. Las evidencias aquí presentes nos permiten destacar el papel fundamental que tiene el estilo de apego seguro en la satisfacción de pareja y sexual.

Las diadas formadas por un hombre seguro y una mujer segura están más satisfechas con sus parejas. Estos datos son acordes con los obtenidos por Ottu y Akpan, (2011). Estas parejas tienen una mayor comunicación, confianza y compromiso; llevan a cabo estrategias adecuadas de resolución de problemas y toma de decisiones; comparten las tareas domésticas, la crianza de los hijos y hay una mayor expresión de afecto y cuidado por parte de ambos miembros.

Las mujeres seguras están menos satisfechas con su pareja cuando él es preocupado. Turan y Vicary (2010) refieren que el apego seguro de las mujeres no actúa como amortiguador de los efectos negativos del apego ansioso de los hombres, es decir, las mujeres pueden mostrar disponibilidad, capacidad de respuesta, confianza, estar dispuestas a desarrollar intimidad y a comunicarse abiertamente pero los hombres ansiosos no lo valoran como tal, no realizan una valoración objetiva de lo que realmente las mujeres seguras le aportan. Y es por ello que ambos se encuentran insatisfechos.

A diferencia de las mujeres, los hombres seguros se encuentran igualmente satisfechos ya sean las mujeres seguras o preocupadas. Esto puede deberse, según Cohn, Silver, Cowan y Pearson (1992), a que el estilo de apego seguro de los hombres actúa como amortiguador de los efectos negativos del apego ansioso de las mujeres.

En resumen, podemos decir que las personas seguras tienen una mayor satisfacción sexual y de pareja que las preocupadas. Las diadas constituidas por un hombre seguro y una mujer segura son las que mayor satisfacción sexual y de pareja tienen. Las mujeres, a diferencia de los hombres, están más satisfechas cuando los dos son seguros, que cuando ella es segura y él ansioso ya que los hombres preocupados no son capaces de valorar el apoyo de una persona segura y las mujeres preocupadas sí. Por tanto, la satisfacción de un miembro de la pareja ansioso/a va a depender del estilo de apego y del sexo de su pareja. Por todo lo cual, parece aconsejable fomentar el desarrollo de un estilo de apego seguro.

Pese a la relevancia de esta investigación este trabajo cuenta con algunas limitaciones. La evaluación a través del uso de autoinformes no siempre es el método más válido para este tipo de procesos, por lo que sería conveniente combinarlo con otro método como la entrevista. Por otro lado, utilizar las redes sociales para acceder a la muestra supone la utilización de una muestra incidental, por lo que sería aconsejable utilizar procedimientos de muestreo que permitan una mayor generalización de las conclusiones. Por último, y como perspectiva futura sería interesante analizar si los resultados obtenidos se mantienen en el tiempo y si también existe un influjo del sistema sexual sobre el sistema de apego, para lo cual sería interesante realizar estudios longitudinales que permitan hacer un seguimiento de la relación entre estos tres constructos y los cambios que se puedan producir con la prolongación de la relación de pareja. 


\section{LA SATISFACCIÓN SEXUAL EN PAREJAS CON ESTILOS DE APEGO SEGURO Y ANSIOSO}

\section{REFERENCIAS}

Alexandrov, E. O., Cowan, P. A. y Cowan, C. P. (2005). Couple attachment and the quality of marital relationships: Method and concept in the validadation of the new couple attachment interview and coding system. Attachment and Human Development, 7(2), 123-152. DOI: 10.1080/14616730500155170.

Armenta, C., Sánchez, R. y Díaz, R. (2014). Exploración e identificación de los determinantes de la satisfacción marital: contexto, individuo e interacción. Revista de Psicología, 10 (19), 7-30.

Banse, R. (2004). Adult attachment and marital satisfaction: evidence for dyadic configuration effects. Journal of Social and Personal Relationships, 21, 273-282. D0I: 10.1177/0265407504041388.

Bartholomew, K. y Horowitz, L. M. (1991). Attachment styles among young adults: A test of a fourcategory model. Journal of Personality and Social Psychology, 61, 224-226. D0I: 10.1037/0022-3514.61.2.226.

Bernier, A. y Dozier, M. (2002). Assesing adult attachment: Empirical sophistication and conceptual bases. Attachment and Human Development, 4(2), 171-179. DOI: 10.1080/14616730210157457.

Birnbaum, G., Reis, H., Mikulincer, M., Gillath, 0. y Orpaz, A. (2006). When Sex Is More Than Just Sex: Attachment Orientations, Sexual Experience, and Relationship Quality. Journal of Personality and Social Psychology, 91 (5), 929-943. D0I:10.1037/0022-3514.91.5.929.

Bowlby, J. (1969/82). Attachment and loss: Vol. 1. Attachment. (2ª ed., 1982). New York: Basic Books.

Bradbury, T. N., Campbell, S. M. y Fincham, F.D. (1995). Longitudinal and behavioral analysis of masculinity and femininity in marriage. Journal of Personality and Social Psychology, 68 (2), 328-341. DOI: 10.1037/0022-3514.68.2.328.

Cohn, D.A., Silver, D.H., Cowan, C.P., Cowan, P.A. y Pearson, J. (1992). Working models of childood attatchment and couple relationship. Journal of Family Issues, 13, 432-449. D0I: 10.1177/019251392013004003.

Fernández-Fuertes, A. A., Orgaz, B., Fuertes, A. y Carcedo, R. (2011). La evaluación del apego romántico en adolescentes españoles: validación de la versión reducida del Experiences in Close Relationships-Revised (ECR-R). Anales de psicología, 27 (3), 827-833.

Fraley, R. C., Waller, N. G. y Brennan, K. A. (2000). An Item Response Theory analysis of self-report measures of adult attachment. Journal of Personality and Social Psychology, 78, 350-365. DOI: 10.1037/0022-3514.78.2.350.

Gómez- Zapiain, J., Ortiz, M.J. y Gómez- Lope, J. (2011). Experiencia sexual, estilos de apego y tipos de cuidados en las relaciones de pareja. Anales de psicología, 27 (2), 447-456.

Gómez-Zapiain, J., Ortiz, M.J. y Gómez-Lope, J. (2012). Capacidad para aportar y solicitar apoyo emocional en las relaciones de pareja en relación con los perfiles de apego. Anales de psicología, 28 (1), 302-312.

Hazan, C. \& Shaver, P. (1987). Romantic love conceptualized as an attachment process. Journal of Personality and Social Psychology, 52, 511-524. DOI: 10.1037/0022-3514.52.3.511.

Hazan, C. y Zeifman, D. (1994). Sex and the psychological tether. En D. Perlman y K. Bartholomew (Eds.), Advances in personal relationships (Vol. 5, pp. 151-180). London: Kingsley.

Holland, A.S., Fraley, R.C. y Roisman, G.I. (2012). Attachment styles in dating couples: Predicting relationship functioning over time. Personal Relationships, 19 (2), 234-246. DOI: 10.1111/j.1475-6811.2011.01350.x.

Lafuente, M. y Cantero, M. (2010). Vinculaciones afectivas: apego, amistad y amor. España: Ediciones Pirámide, S.A. 
MacNeil, S. y Byers, E.S. (1997). The relationship between sexual problems, communication and sexual satisfaction. Canadian Journal of Human Sexuality, 6 (4), 277-283.

MacNeil, S. y Byers, E.S. (2005). Dyadic assessment of sexual self- dosclosure and sexual satisfaction in heterosexual dating couples. Journal of Social and Personal Relationships, 22 (2), 169-181. DOI: 10.1177/026540 7505050942.

Magai, C. (1999). Affect, imagery and attachment. Working models of interpersonal affect and the socialization of emotion. En J. Cassidy y P. R. Shaver (eds.), Handbook of attachment. Theory, research, and clinical aplications (pp.787-802). New York: The Guilford Press.

Martínez- Álvarez, J., Fuertes- Martín, A., Orgaz- Baz, B., Vicario- Molina, I. y González- Ortega, E. (2014). Vínculos afectivos en la infancia y calidad en las relaciones de pareja de jóvenes adultos: el efecto mediador del apego actual. Anales de Psicología, 30 (1), 211-220. DOI: 10.6018/analesps.30.1.135051.

Melero, R. (2008). La relación de pareja, apego, dinámicas de interacción y actitudes amorosas: consecuencias sobre la calidad de la relación (Tesis doctoral, Universidad de Valencia). Recuperado el 2 de Octubre de 2014: http://www.tesisenxarxa.net/TDX-0624109-124526/.

Mikulincer,M., \& Florian, V. (1999). The association between spouses self-reports of attachment styles and representations of family dynamics. Family Process, 38, 69-83. DOI: 10.1111/j.15455300.1999.00069.x

Molero, F., Shaver, P. R., Ferrer, E., Cuadrado, I. y Alonso-Arbiol, I. (2011). Attachment insecurities and interpersonal processes in Spanish couples: A dyadic approach. Personal Relationships, 18 (4), 617-629. DOI: 10.1111/j.1475-6811.2010.01325.x.

Montesi, J.L., Conner, B.T., Gordon, E.A., Fauber, R.L., Kim, K.H. y Heimberg, R.G. (2013). On the Relationship Among Social Anxiety, Intimacy, Sexual Communication, and Sexual Satisfaction in Young Couples. Arch Sex Behav, 42 (1), 81-91. DOI: 10.1007/s10508-012-9929-3.

Ottu, I. F., \& Akpan, U. I. Iboro, F. (2011). Predicting marital satisfaction from the attachment styles and gender of a culturally and religiously homogenous population. Gender \& Behaviour, 9 (1), 3656-3679.

Pick, S. y Andrade, P. (1988a). Desarrollo y validación de la escala de satisfacción marital. Psiquiatría, 4 (1), 9-20.

Rivera, D., Cruz, C. y Muñoz, C. (2011). Satisfacción en las Relaciones de Pareja en la Adultez Emergente: El Rol del Apego, la Intimidad y la Depresión. Terapia Psicológica, 29 (1), 77-83.

Rehman, U. S., Rellini, A. H. y Fallis, E. (2011). The importance of sexual self-disclosure to sexual satisfaction and functioning in committed relationships. Journal of Sexual Medicine, 8, 31083115. DOI: 10.1111/j.1743-6109.2011.02439.x.

Rodríguez, 0. R. (2010). Relación entre satisfacción sexual, ansiedad y prácticas sexuales. Pensamiento psicológico, 7 (14), 41-52.

Schmitt, M., Kliegel, M. y Shapiro, A. (2007). Marital interaction in middle and old age: A predictor of marital satisfaction? The International Journal of Aging \& Human Development, 65 (4), 283300. DOI: 10.2190/AG.65.4.a.

Turan, B. y Vicary, A. M. (2010). Who recognizes and chooses behaviors that are best for a relationship? The separate roles of knowledge, attachment, and motivation. Personality and Social Psychology Bulletin, 36 (1), 119-131. DOI: 10.1177/0146167209349374.

Vera, C. (2010). Satisfacción marital: factores incidentes (Tesis doctoral, Universidad del Bio-Bio de Chile). Recuperado el 20 de febrero de 2015: http://cybertesis.ubiobio.cl/ tesis/2010/vera_c/doc/vera_c.pdf. 
